Cahiers $d u$ MONDE RUSSE

\section{Cahiers du monde russe}

Russie - Empire russe - Union soviétique et États indépendants

$46 / 4 \mid 2005$

L'invention d'une politique humanitaire

\title{
Bruce Adams, Tiny Revolutions in Russia
}

\section{Amandine Regamey}

\section{OpenEdition \\ Journals}

Édition électronique

URL : https://journals.openedition.org/monderusse/6645

DOI : $10.4000 /$ monderusse. 6645

ISSN : $1777-5388$

Éditeur

Éditions de l'EHESS

Édition imprimée

Date de publication : 1 décembre 2005

Pagination : 943-945

ISBN : 2-7132-2057-2

ISSN : $1252-6576$

Référence électronique

Amandine Regamey, "Bruce Adams, Tiny Revolutions in Russia », Cahiers du monde russe [En ligne], 46/4 | 2005, mis en ligne le 29 juin 2009, consulté le 03 septembre 2022. URL : http://

journals.openedition.org/monderusse/6645; DOI : https://doi.org/10.4000/monderusse.6645

Ce document a été généré automatiquement le 3 septembre 2022.

Tous droits réservés 


\title{
Bruce Adams, Tiny Revolutions in Russia
}

\author{
Amandine Regamey
}

\section{RÉFÉRENCE}

Bruce ADAMS, Tiny Revolutions in Russia. Twentieth-Century Soviet and Russian

History in Anecdotes. New York, Londres : RoutledgeCurzon, 2005, 176 p.

1 On ne peut expliquer une plaisanterie sans en perdre le sel, la drôlerie et donc l'intérêt, dit-on généralement. Dans Tiny Revolutions in Russia, Bruce Adams se propose d'inverser ce postulat: une plaisanterie, si elle est expliquée, peut fournir des informations sur la société et la culture dans laquelle elle est née. La plaisanterie constitue en elle-même une explication et les histoires drôles politiques offrent de ce point de vue un matériau de choix pour aborder la société soviétique et l'histoire de la Russie depuis la révolution d'Octobre.

2 Puisant dans plusieurs recueils d'anekdoty publiés à la fin des années 1980, B. Adams passe en revue les thèmes de dérision et les sujets de préoccupation des Soviétiques. Une division chronologique, suivant les dirigeants qui se sont succédé à la tête de l'URSS, conjuguée avec une division thématique à l'intérieur de chaque chapitre, lui permet de présenter les grands cycles d'histoires drôles politiques.

3 S'il existait peu d'histoires drôles sur Lenin de son vivant, leur nombre explose en revanche dans les années 1970. Plus que Lenin lui-même, ce sont les extravagances du culte et en particulier la célébration du centenaire de sa naissance qui sont tournées en dérision. Inversant l'image officielle, les anekdoty le présentent en mari trompé, trahi par son fidèle acolyte Dzeržinskij, se moquent des souvenirs officiels, contredisent l'idée de sa «bonté » et inventent des " produits dérivés » du culte, tel le soutien-gorge « Monts Lenin ».

4 Très différentes, les histoires sur Stalin, qui, elles, circulaient de son vivant, insistent sur les purges qui ont marqué son règne, la terreur et les répressions aveugles (y 
compris contre les colporteurs d'histoires drôles). Elles le présentent comme un tyran sanguinaire, mais s'attachent aussi à dénoncer le culte de la personnalité.

5 L'hypocrisie de la déstalinisation lancée par Hruščev inaugure le cycle des blagues sur ce dirigeant brocardé pour sa calvitie et son physique porcin. Sa politique brouillonne, le but proclamé de construire le communisme en vingt ans et de l'« exporter » dans le reste du monde, ainsi que la compétition avec les États-Unis sont les thèmes privilégiés des anekdoty, qui suggèrent que l'avènement du communisme ne ferait que décupler les difficultés de l'URSS et entraînerait une véritable catastrophe pour les pays alentours.

6 Sous Brežnev, les difficultés, les pénuries et l'essoufflement des grands projets, tel que le chantier ferroviaire du BAM, restent d'actualité. Mais c'est surtout de l'incapacité de Brežnev à lire un discours que l'on se moque, ainsi que de son goût pour le clinquant et les décorations, et du culte de la personnalité qu'il laisse s'instaurer (ou plutôt « du culte, car on cherche toujours la personnalité... »). Les histoires drôles sur un Brežnev sénile à la fin de sa vie trouvent un écho dans celles qui circulent sur ses deux successeurs, Andropov ou Černenko, même si le premier, homme du KGB, y apparaît aussi sous les traits d'un véritable garde-chiourme.

7 Quant à Gorbačev, c'est sa politique économique, mais surtout sa politique antialcoolique, qui sont ciblées, de même que la place inhabituelle tenue par sa femme, Rajsa Maksimovna.

8 Les anekdoty évoquent les grands moments de l'histoire de l'URSS : la période de chaos qui suit la révolution de 1917, l'arrivée au pouvoir de Stalin, les purges, la collectivisation et le premier plan quinquennal, la déstalinisation, la longue période de stagnation brejnévienne, la succession des premiers secrétaires séniles au début des années 1980, la perestroïka et la catastrophe de Tchernobyl. À noter, comme le fait B. Adams, que la «Grande Guerre patriotique » de 1941-1945 est quasiment absente du comique soviétique.

9 Malgré la présentation chronologique que propose l'auteur, un certain nombre de thèmes transversaux peuvent être repérés. Celui des interventions extérieures de l'URSS tout d'abord, de la guerre de Corée à la Tchécoslovaquie en 1968, en passant par l'insurrection hongroise de 1956 (B. Adams n'évoque pas l'Afghanistan, pourtant sujet d'histoires puisque si l'URSS l'a envahi, « c'est qu'elle procède par ordre alphabétique »). La crainte de la puissance de la Chine avec laquelle les relations se dégradent progressivement transparaît à toutes les époques.

10 Les problèmes économiques, les dysfonctionnements de l'agriculture, les pénuries reviennent comme un leitmotiv, de même que l'omniprésence de la propagande, l'absence de liberté d'expression et l'aspiration de la majorité des Soviétiques à quitter le pays. Le personnage de Rabinovič apparaît dès les années 1920 et partage avec les histoires de la radio arménienne le rôle de critique attitré et plein d'esprit du système soviétique. Mais le thème de l'antisémitisme ou celui de l'émigration massive des Juifs dans les années 1970 sont également au cœur de nombreuses histoires, peut-être, comme le suggère l'auteur, parce qu'elles étaient racontées essentiellement par l'intelligentsia juive.

11 Les grandes séries d'anekdoty sur le commandant rouge Čapaev et l'agent soviétique en Allemagne nazie Štirlic, toutes deux inspirées de films, occupent dans le livre une place à la hauteur de leur popularité, tout comme les histoires sur certaines nationalités, tels les 
Tchouktches (pour leur bêtise non exempte de malice) ou les Géorgiens (habiles à tirer profit du système).

Enfin, les avantages dont bénéficie la nomenklatura et surtout son manque d'éducation, défaut auquel n'échappe pas même la ministre de la Culture Furceva, sont les thèmes privilégiés de nombreuses histoires drôles. Ces critiques se poursuivront d'ailleurs dans les blagues sur les Nouveaux Russes, apparues dans les années 1990, qui semblent clore la tradition des histoires drôles politiques.

13 On peut néanmoins regretter que ces grands thèmes soient dispersés tout au long du livre, et le choix fait par l'auteur de classer les histoires en fonction des premiers secrétaires du parti présente de ce point de vue des limites certaines. Soulignant l'intervalle qu'il peut y avoir entre un événement et son reflet dans les histoires drôles (la révolution et la guerre civile n'ont donné lieu que bien plus tard à des blagues se moquant de la manière dont ces événements étaient enseignés), il classe les anekdoty à la période où elles sont apparues et non pas à la période à laquelle elles se réfèrent. C'est ainsi que la majorité des blagues sur Lenin se retrouve dans la partie intitulée «Brežnev » - puisque effectivement elles ont fleuri à l'époque de la stagnation.

Alors que la lecture en est rendue moins fluide, il n'est pas sûr que B. Adams évite pour autant le piège de la fausse datation. De plus, en mettant l'accent sur la période où elles ont émergé plutôt que sur leur contenu, il se prive (et nous prive aussi) de voir comment ces histoires ont évolué selon les périodes, alors que cette évolution pourrait en apprendre beaucoup sur leur signification. Il nous prive également d'une approche transversale, qui aurait sans doute permis de dégager, au-delà des fluctuations historiques, les lignes de force et les points centraux de ce que signifiait «être soviétique ».

On aborde là sans doute la principale lacune du livre. Tiny Revolutions se présente comme un manuel pour les étudiants en " culture et histoire russe et soviétique ", et il en adopte d'ailleurs le ton. Mais les éclaircissements que B. Adams fournit se limitent au contenu des histoires drôles et à leur contexte : il n'explique en définitive que très peu ce qu'elles signifiaient pour ceux qui les racontaient.

Ces anekdoty étaient-elles vraiment, comme le suggère le titre du livre, des « révolutions en miniature », de celles qui, comme l'écrivait Orwell, font rire car elles sapent la dignité des puissants? Ceux qui les racontaient concevaient-ils ce genre comme une protestation, une forme d'engagement civique qui aurait pris d'autres aspects dans un régime démocratique, comme le suggère l'auteur à plusieurs reprises ? La disparition de ces histoires à la fin des années 1990 tient-elle uniquement à ce qu'il est désormais possible de s'exprimer en toute liberté et au fait que l'intelligentsia qui les colportait se heurte après la chute de l'URSS à des difficultés beaucoup trop importantes pour avoir envie d'en rire? Ou n'est-ce pas un signe de perte de familiarité avec le régime, perte de cette sécurité qu'offrait le fait d'être citoyen de l'une des deux superpuissances? L'anekdot n'était-elle pas aussi un moyen d'échanger entre amis et connaissances des "signes de complicité ", le partage d'une même attitude envers le pouvoir, et n'est-ce pas avec la disparition des histoires drôles politiques un mode de sociabilité qui s'est perdu ? À défaut de répondre à ces questions, ce livre n'en reste pas moins un recueil très complet, qui brosse un tableau vaste et drôle de l'histoire de la Russie soviétique. 